\title{
INDICADORES GERENCIAIS DO MAPA CIRÚRGICO DE UM HOSPITAL UNIVERSITÁRIO
}

\author{
Management indicators of the surgical schedule in a university hospital \\ Indicadores de gestión del mapa quirúrgico de un hospital universitario \\ Débora Oliveira Nunes da Silva Reis ${ }^{1 *}$ (D), Ricardo de Oliveira Meneses $^{2}$ (D), \\ Cecília Maria Izidoro Pinto ${ }^{3}$ D, Maria Virgínia Godoy da Silva ${ }^{4}$ D, Nathália Ferreira Teixeira ${ }^{5}$
}

RESUMO: Objetivos: Analisar a produtividade cirúrgica de um hospital universitário relacionando os indicadores gerenciais do mapa cirúrgico com os aspectos do planejamento e propor estratégias para elaboração do mapa e agendamento cirúrgico. Método: Trata-se de uma pesquisa quantiqualitativa, descritiva, documental, transversal, realizada no centro cirúrgico de um hospital universitário, de abril a junho de 2018, por meio da análise dos mapas cirúrgicos. Resultados: Analisou-se no trimestre uma média de 400 cirurgias mensais. Identificou-se que as cirurgias de porte II (55,9\%) foram as mais frequentes, sendo a urologia (18,7\%) a especialidade mais recorrente. A taxa de cancelamento avaliou o índice de desempenho do planejamento, que foi de 16,9\%. Conclusões: A produtividade cirúrgica e a taxa de cancelamento encontrada aproximam-se da realidade de outros hospitais universitários do país, e as fragilidades apontadas são pertinentes às falhas no planejamento, gerando custos institucionais. Recomenda-se, como estratégia de planejamento, a realização do bate-mapa, a visita pré-operatória de enfermagem e a confirmação do paciente. Palavras-chave: Enfermagem de Centro Cirúrgico. Gestão em saúde. Planejamento hospitalar. Hospitais universitários.

ABSTRACT: Objectives: To analyze the surgical productivity in a university hospital, correlating the management indicators of the surgery schedule with planning aspects, and propose strategies for developing the surgery schedule. Method: This is a quantitative and qualitative study of descriptive, documentary, and cross-sectional nature, performed at the surgical center of a university hospital, from April to June 2018, based on the analysis of surgery schedules. Results: In the quarter, an average of 400 monthly surgeries were assessed. We identified that magnitude II (55.9\%) surgeries were the most frequent, and urology (18.7\%) was the predominant specialty. The cancellation rate evaluated the planning performance index, which was $16.9 \%$. Conclusions: The surgical productivity and the cancellation rate found were close to those of other university hospitals in the country, and the weaknesses detected are related to planning failures, leading to institutional costs. We recommend the implementation of a schedule review, a preoperative nursing visit, and patient confirmation as a planning strategy. Keywords: Operating room nursing. Health management. Hospital planning. Hospitals, university.

RESUMEN: Objetivos: Analizar la productividad quirúrgica de un hospital universitario contra los indicadores de gestión del mapa quirúrgico con los aspectos de planificación y proponer estrategias para la elaboración del mapa y el calendario quirúrgico. Método: Esta es una investigación cuantitativa, cualitativa, descriptiva, documental, transversal, realizada en el quirófano de un hospital universitario, de abril a junio de 2018, a través del análisis de mapas quirúrgicos. Resultados: Se analizó un promedio de 400 cirugías mensuales durante el trimestre. Se encontró que las cirugías de tamaño II (55.9\%) fueron las más frecuentes, siendo la urología (18.7\%) la especialidad más recurrente. La tasa de cancelación evaluó el índice de desempeño de planificación, que fue de 16.9\%. Conclusiones: la productividad quirúrgica y la tasa de cancelación están cerca de la realidad de otros hospitales universitarios en el país y las debilidades identificadas son pertinentes a las fallas de planificación, generando costos institucionales. Como estrategia de planificación, se recomienda realizar el toque de mapa, la visita de enfermería preoperatoria y la confirmación del paciente. Palabras clave: Enfermería de quirófano. Gestión en salud. Planificación hospitalaria. Hospitales universitarios.

\footnotetext{
'Enfermeira da cirurgia vascular na Policlínica Piquet Carneiro, da Universidade do Estado do Rio de Janeiro (UERJ); pós-graduada pelo Programa de Residência em Enfermagem em Centro Cirúrgico e Centro de Material e Esterilização da UERJ - Rio de Janeiro (RJ), Brasil.

2Professor assistente do Programa de Residência em Enfermagem em Centro Cirúrgico e Centro de Material e Esterilização, da UERJ; mestre pela UERJ - Rio de Janeiro (RJ), Brasil.

${ }^{3}$ Professora da graduação em Enfermagem no Hospital Universitário Clementino Fraga, da Universidade Federal do Rio de Janeiro (UFRJ); doutora pela UFRJ - Rio de Janeiro (RJ), Brasil.

4Professora do curso de Enfermagem da Universidade Veiga de Almeida; Doutora em Enfermagem pela Escola de Enfermagem da USP - São Paulo (SP), Brasil.

${ }^{5}$ Enfermeira de cirurgia robótica no Centro de Material e Esterilização do Hospital Universitário Pedro Ernesto, da UERJ; pós-graduada na modalidade Residência em Enfermagem em Centro Cirúrgico e Centro de Material e Esterilização da UERJ - Rio de Janeiro (RJ), Brasil.

*Autor correspondente: dbreis13@gmail.com

Recebido: 03/02/2019 - Aprovado: 22/09/2019

DOI: $10.5327 / Z 1414-4425201900040007$
} 


\section{INTRODUÇÃO}

O planejamento dos serviços de assistência perioperatória demanda um fluxo de informações capazes de articular alguns setores e serviços. Para garantir boa produtividade cirúrgica, alguns aspectos são necessários, tais como: adequação da estrutura física, dimensionamento de recursos humanos, previsão e provisão de recursos materiais e equipamentos e apoio dos serviços que atuam direta ou indiretamente na execução dos procedimentos cirúrgicos ${ }^{1}$.

Nesse contexto, a Unidade de Centro Cirúrgico (CC) destaca-se pela complexidade técnica, processual e multidisciplinar, pois envolve um conjunto de elementos destinados à realização de procedimentos anestésico-cirúrgicos, bem como a recuperação do paciente ${ }^{2,4}$.

Para a execução desses procedimentos cirúrgicos, são essenciais integralidade e articulação dos serviços de internação, almoxarifado, farmácia, banco de sangue, laboratório, radiologia, centro de material e esterilização, lavanderia, engenharia clínica, unidades de terapia intensiva, além das órteses, próteses e materiais especiais.

O mapa cirúrgico é um instrumento fundamental desse planejamento, pois por meio dele é possível dimensionar as salas cirúrgicas e os procedimentos agendados, além de trazer dados dos pacientes, como nome, idade, registro, origem, cirurgia a ser realizada, porte cirúrgico, equipes cirúrgicas, anestesiologistas, pessoal de enfermagem e serviços de apoio imprescindíveis 5 .

Considera-se o enfermeiro perioperatório um profissional habilitado para gerenciar os procedimentos anestésico-cirúrgicos, porque demanda formação específica e é responsável pelas intervenções de enfermagem. Ainda, ele atua na prevenção de complicações decorrentes de falhas no planejamento. O melhor gestor é aquele que conhece profundamente as atividades desenvolvidas na sua unidade ${ }^{2,3,6}$.

A eficiência do CC pode ser representada quantitativamente pelos resultados, situações, ocorrências e eventos relacionados ao seu funcionamento. Os indicadores de qualidade constituem elemento fundamental para a tomada de decisão, já que por eles é possível monitorar, com maior eficácia, os eventos e fornecer informações para medidas de correções e padronizações. Esses dados compõem uma estratégia gerencial para avaliação da produtividade ${ }^{7}$.

Esta pesquisa justifica-se pelos aspectos gerencias na análise do mapa cirúrgico, trazendo repercussões diretamente ligadas à segurança do paciente na realização dos procedimentos cirúrgicos, especialmente na realidade de um hospital universitário (HU). Sendo assim, formularam-se as questões da pesquisa: por meio do mapa cirúrgico, quais indicadores são gerados como resultado do planejamento? Que estratégias devem ser adotadas adiante da produtividade cirúrgica?

\section{OBJETIVOS}

Analisar a produtividade cirúrgica de um HU, por meio de indicadores do mapa cirúrgico, e elaborar estratégias para o planejamento do agendamento cirúrgico.

\section{MÉTODO}

Trata-se de uma pesquisa quantiqualitativa, descritiva, documental e transversal realizada no CC de um HU de alta complexidade no estado do Rio de Janeiro, que se destaca como campo de treinamento, ensino e pesquisa.

O recorte temporal abrangeu o período de abril a junho de 2018, estando o CC com 10 salas operatórias (SO) em funcionamento, com média de 400 cirurgias mensais.

O mapa cirúrgico era composto de cirurgias eletivas feitas nos períodos diurno e vespertino, de segunda a sexta-feira, com menos quantidade aos sábados.

O planejamento do mapa funciona com uma agenda prévia das especialidades cirúrgicas, dispostas pelos dias da semana entre os turnos para distribuição das SO.

Para a confecção do mapa, foram utilizados informativos impressos e preenchidos manualmente, no qual se encontram as informações sobre o paciente, o procedimento, os serviços de apoio e a equipe cirúrgica.

As especialidades ficam responsáveis pela verificação da disponibilidade de leitos para internação, assim como dos serviços de apoio necessários para o procedimento. O protótipo do mapa é direcionado à chefia médica, que o encaminha para os anestesiologistas e enfermeiros, a fim de comporem as equipes para cada SO.

A análise documental desenvolveu-se por meio de um roteiro semiestruturado, pela apreciação do mapa cirúrgico, dos impressos de solicitação de urgência, da folha de recepção do paciente e pelo livro de ordens e ocorrências dos enfermeiros do CC.

Para transcrever os dados qualitativos, a observação em campo teve como principal objetivo registrar as informações pertinentes ao fluxo do mapa.

Os critérios de elegibilidade envolveram as cirurgias que se encontravam no mapa cirúrgico, incluindo as de urgências e as cirurgias realizadas por meio de projetos que recebem 
financiamento externo via Ministério da Saúde, fundo de pesquisa ou de empresa privada.

Foram excluídas as cirurgias oftalmológicas, as da endourologia e as demais que não foram realizadas nas dependências do CC.

Este estudo cumpriu as normas da Resolução n ${ }^{\circ}$ 466/2012, do Conselho Nacional de Saúde, e o projeto foi aprovado pelo Comitê de Ética em Pesquisa do HU Pedro Ernesto, recebendo parecer favorável protocolado (Certificado de Apresentação para Apreciação Ética — CAAE 82754217.6.0000.5259).

\section{RESULTADOS}

No período de abril a junho de 2018, planejaram-se 1.481 cirurgias no $\mathrm{HU}$, das quais foram canceladas 282. A Tabela 1 mostra que houve 186 solicitações de cirurgia em caráter de urgência. Das cirurgias programadas no mapa cirúrgico, 1.079 correspondem àquelas sem o financiamento dos projetos e 402 por meio dos projetos.

Analisou-se que, no período estudado, a média trimestral era de 400 cirurgias por mês, o que permitia taxa de 1,3 cirurgia por SO por dia.

As cirurgias eletivas feitas sem o financiamento oriundo de projetos foram contabilizadas em $81,1 \%(n=875)$, e as eletivas com projetos, em $88,1 \%(n=354)$. Entre as solicitações de urgências, foram realizadas $83,9 \%(n=156)$ cirurgias.

A taxa de cancelamento global foi de $16,9 \%(n=282)$. Entre os cancelamentos, as cirurgias sem projetos obtiveram frequência de $18,9 \%(n=204)$, enquanto as com projetos contabilizaram $11,9 \%(n=48)$ e a frequência daquelas de urgências foi de $16,1 \%(n=30)$.

As especialidades médicas foram organizadas de acordo com o porte cirúrgico para as cirurgias eletivas realizadas, segundo classificação do tempo cirúrgico, em que se adotou porte I para cirurgias de até 2 horas de duração, porte II de 2 a 4 horas, porte III de 4 a 6 horas e porte IV acima de 6 horas ${ }^{1}$.

A urologia representou $14,4 \%(n=177)$ das cirurgias de porte II e $2,8 \%(n=35)$ porte III. A cirurgia geral apresentou destaque para os procedimentos porte II, com $6,7 \%(n=82)$ e 2,0\% $(\mathrm{n}=25)$ para porte I. A cirurgia pediátrica teve $5,6 \%$ $(n=69)$ de cirurgias porte II e $2,7 \%(n=33)$ porte I.

A maioria das especialidades apresentou maiores frequências em cirurgias de portes I, II e III, contudo a cirurgia cardíaca e a neurocirurgia predominaram nas cirurgias de porte IV, com 6,7\% $(n=82)$ e $2,4 \%(n=29)$, respectivamente (Tabela 2$)$.

Os serviços de apoio solicitados no mapa cirúrgico foram raio $\mathrm{X}$; banco de sangue; unidade de terapia intensiva; intensificador, que permite imagens em alta resolução; e videolaparoscópio.

O videolaparoscópio apresentou maior frequência - 30,8\% $(\mathrm{n}=472)$ - , seguido do banco de sangue, $26,4 \%(\mathrm{n}=398)$, e do serviço de imagem pelo uso do arco em C - 23,8\% $(\mathrm{n}=293)$. Os serviços de menos requisições em SO foram raio $\mathrm{X}-21,8 \%(\mathrm{n}=272)$, unidade de terapia intensiva $-15,7 \%$ $(\mathrm{n}=234)$ e o laboratório $-7,1 \%(\mathrm{n}=113)$, conforme Tabela 3.

As especialidades cirúrgicas com mais solicitações de urgência foram a cirurgia geral, $\operatorname{com} 22,6 \%(n=42)$; seguida da urologia, com $19,9 \%(n=37)$; neurocirurgia, $10,2 \%(n=19)$; cardíaca, $9,1 \%(n=17)$; vascular, $9,1 \%(n=17)$; e cirurgia torácica, $7,5 \%(n=14)$ (Tabela 4).

A taxa de cancelamento global foi de $16,9 \%(n=282)$ entre as cirurgias eletivas e de urgência. As principais causas foram a falta de sangue, com 13,5\% $(n=38)$; o adiantado da hora, $12,4 \%(n=35)$; pacientes sem condições clínicas, $11,3 \%$ $(\mathrm{n}=32)$; não internação do paciente, 9,9\% $(\mathrm{n}=28)$; stand by, $9,9 \%(n=28)$; falta de vaga em unidade de terapia intensiva, $8,5 \%(n=24)$; pacientes sem exames, $7,8 \%(n=22)$; falta de material/ equipamento, $6,4 \%(n=18)$. Obtiveram-se menores frequências para o paciente que se alimentou $-6 \%(n=17)$; outros - 5,7\% $(n=16)$; em branco - 3,5\% $(n=10)$; ausência de staff médico - 3,2\% ( $\mathrm{n}=9)$; e mudança de conduta terapêutica, com $1,8 \%(\mathrm{n}=5)$, conforme Tabela 5.

\section{DISCUSSÃO}

Usando alguns indicadores de processos no CC, pode-se avaliar a qualidade dos serviços na assistência perioperatória por meio da mensuração dos processos gerenciais, da taxa

Tabela 1. Produção de cirurgias agendadas, de urgência e canceladas, com e sem projetos, de abril a junho de 2018.

\begin{tabular}{|l|c|c|c|c|c|c|}
\multirow{2}{*}{ Cirurgias } & \multicolumn{2}{|c|}{ Agendadas/Solicitadas } & \multicolumn{2}{|c|}{ Realizadas } & \multicolumn{2}{c|}{ Canceladas } \\
\cline { 2 - 7 } & $\mathbf{n}$ & $\%$ & $\mathbf{n}$ & $\%$ & $\mathbf{n}$ & 18,9 \\
\hline Eletivas sem projetos & 1.079 & 64,7 & 875 & 81,1 & 204 & 11,9 \\
\hline Eletivas com projetos & 402 & 24,1 & 354 & 88,1 & 48 & 16,1 \\
\hline Urgências & 186 & 11,2 & 156 & 83,9 & 282 & 16,9 \\
\hline Total & 1.667 & 100,0 & 1.385 & 83,1 & & 30 \\
\hline
\end{tabular}


de produtividade e da taxa de cancelamento de cirurgias. Dos aspectos observados na taxa de produtividade, analisaram-se os relacionados à taxa de utilização das $\mathrm{SO}$ por dia $\mathrm{e}$ ao número de cirurgias por mês e por equipes².

A produtividade cirúrgica considerada no trimestre para as cirurgias eletivas e de urgência se aproxima dos resultados da pesquisa realizada no HU em Minas Gerais, com uma amostra de 1.895 cirurgias, sendo 1.542 eletivas e 353 de urgência, conotando uma característica dos $\mathrm{HU}$ para atendimentos eletivos ${ }^{8}$.

No presente estudo, a taxa de cancelamento observada (16,9\%) aproxima-se quando equiparada às dos HU de Sergipe e Paraná, que obtiveram taxas de cancelamento de 19,5 e 18,45\%, respectivamente. No entanto, no estudo de um HU no sul de Minas Gerais, pôde-se observar frequência de $27,4 \%$ e em um hospital de Pernambuco de 30,6\%, quase o dobro do apurado aqui ${ }^{9,12}$.

Todavia, apesar de a média nacional levantada ser próxima à taxa de cancelamento do HU, outro hospital-escola público no interior de São Paulo alcançou 6,79\%, resultado este vinculado à realização da visita pré-operatória ${ }^{13}$.

Considera-se que as causas mais frequentes do cancelamento das cirurgias, neste estudo, estão ligadas à ineficiência do planejamento, condicionada às questões evitáveis, como a falta de sangue e o adiantado da hora ${ }^{14}$.

A falha na comunicação no CC entre seus usuários e os serviços de apoio associada à baixa nos estoques do banco de sangue e ao fato de os pacientes internarem menos de
24 horas antes do procedimento dificultou a identificação e a disponibilidade de fluidos, influenciando nos resultados ${ }^{14}$.

O mapa cirúrgico é o documento que possibilita a execução do fluxo de atendimento dos pacientes eletivos no setor. Um sistema de agendamento efetivo favorece o trabalho de cirurgiões, anestesiologistas, profissionais de enfermagem, equipamentos e das $\mathrm{SO}^{15,16}$.

A condição clínica do paciente é uma variável interdependente no cancelamento das cirurgias, pois, como estas são agendadas pelos médicos, ocorrem alterações no quadro clínico de alguns pacientes, pelas doenças crônicas descompensadas, como hipertensão, hipoglicemia, infecções e outras afecções ${ }^{8}$.

Tabela 3. Solicitação dos serviços de apoio ao mapa cirúrgico, entre abril e junho de 2018.

\begin{tabular}{|l|c|c|}
\hline Serviços de apoio & $\begin{array}{c}\text { Solicitação no } \\
\text { mapa cirúrgico } \\
\text { n }\end{array}$ & $\%$ \\
\hline Videolaparoscópio & 472 & 30,8 \\
\hline Banco de sangue & 398 & 26,4 \\
\hline $\begin{array}{l}\text { Intensificador de imagem (arco em } \\
\text { C) }\end{array}$ & 293 & 23,8 \\
\hline Raios X & 272 & 21,8 \\
\hline Unidade de terapia intensiva & 234 & 15,7 \\
\hline Laboratório para congelação & 113 & 7,1 \\
\hline
\end{tabular}

Tabela 2. Produtividade cirúrgica por especialidades e porte cirúrgico, entre abril e junho de 2018.

\begin{tabular}{|l|c|c|c|c|c|c|c|c|c|c|}
\hline \multirow{2}{*}{ Especialidade cirúrgica } & \multicolumn{2}{|c|}{ Porte I } & \multicolumn{2}{|c|}{ Porte II } & \multicolumn{2}{c|}{ Porte II } & \multicolumn{2}{c|}{ Porte IV } & \multicolumn{2}{c|}{ Ranking por especialidades } \\
\cline { 2 - 8 } & $\mathbf{n}$ & $\mathbf{\%}$ & $\mathbf{n}$ & $\mathbf{\%}$ & $\mathbf{n}$ & $\mathbf{\%}$ & $\mathbf{n}$ & $\mathbf{\%}$ & $\mathbf{n}$ & $\%$ \\
\hline Urologia & 18 & 1,5 & 177 & 14,4 & 35 & 2,8 & - & - & 230 & 18,7 \\
\hline Geral & 25 & 2,0 & 82 & 6,7 & 18 & 1,5 & - & - & 125 & 10,2 \\
\hline Pediatria & 33 & 2,7 & 69 & 5,6 & 9 & 0,7 & - & - & 111 & 9,0 \\
\hline Plástica & 23 & 1,9 & 54 & 4,4 & 31 & 2,5 & - & - & 108 & 8,8 \\
\hline Cardíaca & - & - & 3 & 0,2 & 18 & 1,5 & 82 & 6,7 & 103 & 8,4 \\
\hline Neurologia & 12 & 1,0 & 27 & 2,2 & 33 & 2,7 & 29 & 2,4 & 101 & 8,2 \\
\hline Proctologia & 18 & 1,5 & 52 & 4,2 & 12 & 1,0 & - & - & 82 & 6,7 \\
\hline Otorrinolaringologia & 09 & 0,7 & 27 & 2,2 & 46 & 3,7 & - & - & 82 & 6,7 \\
\hline Ortopedia & 14 & 1,1 & 42 & 3,4 & 10 & 0,8 & - & - & 66 & 5,4 \\
\hline Ginecologia & 7 & 0,6 & 54 & 4,4 & 03 & 0,2 & - & - & 64 & 5,2 \\
\hline Tórax & 17 & 1,4 & 32 & 2,6 & 12 & 1,0 & - & - & 61 & 5,0 \\
\hline Vascular & 12 & 1,0 & 29 & 2,4 & 05 & 0,4 & - & - & 46 & 3,7 \\
\hline Bucomaxilofacial & - & - & 27 & 2,2 & 03 & 0,2 & - & - & 30 & 2,4 \\
\hline Anomalias craniofaciais & 8 & 0,7 & 12 & 1,0 & - & - & - & - & 20 & 1,6 \\
\hline Total & 196 & 16,1 & 687 & 55,9 & 235 & 19,1 & 111 & 9,0 & 1.229 & 100,00 \\
\hline
\end{tabular}


Os resultados encontrados para essa problemática foram apontados em outras realidades, como no HU de Minas Gerais, com $19,1 \%$, e no Recife, com $20,8 \% \%^{8,12}$.

A visita pré-operatória é uma estratégia na identificação precoce desses problemas relacionados às condições clínicas

Tabela 4. Solicitação de urgências por especialidades, de abril a junho de 2018.

\begin{tabular}{|l|c|c|}
\hline $\begin{array}{l}\text { Solicitação de urgência por } \\
\text { especialidade }\end{array}$ & $\mathbf{n}$ & $\%$ \\
\hline Geral & 42 & 22,6 \\
\hline Urologia & 37 & 19,9 \\
\hline Neurologia & 19 & 10,2 \\
\hline Cardíaca & 17 & 9,1 \\
\hline Vascular & 17 & 9,1 \\
\hline Tórax & 14 & 7,5 \\
\hline Proctologia & 9 & 4,8 \\
\hline Pediatria & 8 & 4,3 \\
\hline Otorrinolaringologia & 7 & 3,8 \\
\hline Centro Obstétrico & 5 & 2,7 \\
\hline Plástica & 5 & 2,7 \\
\hline Ginecologia & 2 & 1,1 \\
\hline Anomalias craniofaciais & 2 & 1,1 \\
\hline Ortopedia & 2 & 1,1 \\
\hline Total & 186 & 100,0 \\
\hline
\end{tabular}

Tabela 5. Causas de cancelamentos de cirurgias, entre abril e junho de 2018.

\begin{tabular}{|l|c|c|}
\hline Causas da suspensão & $\mathbf{n}$ & $\%$ \\
\hline Falta de sangue & 38 & 13,5 \\
\hline Adiantado da hora & 35 & 12,4 \\
\hline Paciente sem condições clínicas & 32 & 11,3 \\
\hline Paciente não internou & 28 & 9,9 \\
\hline Stand by & 28 & 9,9 \\
\hline Falta de vaga na unidade de terapia & 24 & 8,5 \\
\hline intensiva & 22 & 7,8 \\
\hline Paciente sem exames & 18 & 6,4 \\
\hline Falta de material/equipamento & 17 & 6,0 \\
\hline Paciente alimentou-se & 16 & 5,7 \\
\hline Outros & 10 & 3,5 \\
\hline Em branco & 9 & 3,2 \\
\hline Ausência de staff médico & 5 & 1,8 \\
\hline Mudança de conduta terapêutica & 282 & 100,0 \\
\hline Total & & \\
\hline
\end{tabular}

do paciente, porque evita a alocação desse paciente no mapa e, por conseguinte, o cancelamento ${ }^{11}$.

A não internação do paciente também é outro fator apresentado pelo hospital do Recife (24,3\%). A prática de ligar para os pacientes na véspera da cirurgia foi uma estratégia que diminuiu a taxa de não internação $(8,96 \%)$, como revela uma pesquisa realizada em um HU no interior de São Paulo ${ }^{12,13}$.

Os cancelamentos trazem inúmeros prejuízos para o hospital, uma vez que o planejamento é realizado na preparação da logística de materiais, equipamentos, instrumentais e equipes específicas para o procedimento. Os hospitais públicos são reembolsados pelo Sistema Único de Saúde (SUS), com base em uma tabela única, com valores para cada procedimento executado, e esses valores reembolsados independem do tempo de permanência do paciente no hospital ou de seus reais custos hospitalares ${ }^{8,17}$.

Um estudo realizado num hospital de grande porte em São Paulo, aprovado pela Organização Nacional de Acreditação(ONA) e reconhecido pela Joint Commission International (JCI) apresentou a estratégia do bate-mapa como ferramenta de gestão de qualidade na redução de cancelamentos e atrasos de cirurgias ${ }^{18}$.

Percebeu-se que a falha em algum dos processos de trabalho, além de comprometer a segurança do paciente, pode acarretar no atraso e até mesmo no cancelamento de cirurgias ${ }^{16}$.

Neste estudo foi elaborado o diagrama de Ishikawa para mapeamento das fragilidades, no qual foi identificada uma série de fatores, sendo o maior deles a falta de produtos para atender às demandas das cirurgias eletivas, de urgência e de emergência ${ }^{18}$.

Após identificação e análise dos problemas, foram instituídas três frentes de ações para solucionar a problemática. Uma delas é o bate-mapa, que se configura como reuniões diárias dos integrantes interprofissionais ligados à assistência perioperatória, desde setores do CC, centro de materiais, internação, engenharia clínica e outros, para identificar e minimizar as fragilidades do serviço e propor melhorias para assistência ao paciente cirúrgico ${ }^{18}$. A distribuição das cirurgias por porte é importante para o dimensionamento do pessoal de enfermagem, pois essa classificação permite que a SO seja organizada para ser atendida conforme a programação diurna do mapa operatório, considerando tempo de limpeza e montagem de sala entre as cirurgias ${ }^{1,5}$.

O maior quantitativo de cirurgias foi porte II ( $55,9 \%)$. Esse tipo de cirurgia tem sua duração de 2 a 4 horas, composta, na sua maioria, da especialidade da urologia, justificada pela realização do projeto. As de porte III $(19,1 \%)$ tiveram duração de 4 a 6 horas 5 .

Estudo realizado no HU de Cascavel, no Paraná, obteve taxa de cirurgias porte I, porte II, porte III e porte IV de 64, 31, 4 e $1 \%$, respectivamente. As características dos atendimentos 
do hospital interferem diretamente na realização do procedimento e no seu porte, isto é, determinam o planejamento e os resultados alcançados ${ }^{19}$.

Outro aspecto importante é a avaliação dos processos de apoio que influenciam na qualidade gerencial do CC, pois são levadas em consideração a área crítica e restrita e a sua necessidade de integração, de modo a trazerem dinâmica organizacional ao agendamento cirúrgico ${ }^{20,21}$.

Entre esses serviços, as cirurgias videolaparoscópicas são uma alternativa minimamente invasiva em relação à cirurgia convencional e obtiveram mais solicitações no mapa cirúrgico analisado.

Puderam-se considerar, para este estudo, o banco de sangue, o uso de intensificadores de imagem e raio- $\mathrm{X}$, a unidade de terapia intensiva e o laboratório de análises clínicas como facilitadores do procedimento cirúrgico na otimização do tempo no planejamento do mapa cirúrgico.

Observou-se, ainda, que o número de solicitações de urgência foi acrescido pela substituição de alguns pacientes que, em decorrência de alguns fatores, não estavam relacionados no agendamento.

A cirurgia geral foi a especialidade mais recorrente nos pedidos de urgência no período do estudo, dado justificado pelo atendimento ao plantão geral. Alguns pacientes que precisaram de atendimento de urgência após o período diurno eram atendidos pela cirurgia geral, sendo o motivo dos dados deste estudo, acrescendo-se o serviço de urologia, por conter um projeto institucional.

Ressalta-se a falta de participação do enfermeiro no planejamento do CC, na alocação dos procedimentos em SO, na provisão e previsão de materiais e equipamentos, na articulação com os serviços de apoio, com vistas à segurança do paciente, à melhor eficiência do CC e a práticas que expressem os resultados dos seus próprios processos e que orientem o monitoramento, a mensuração e a avaliação da qualidade ${ }^{2}$.

O enfermeiro é o profissional com autonomia e articulação entre as equipes e serviços para monitorar e realizar as intervenções que julgue necessárias no agendamento cirúrgico, para melhor gerenciamento e controle dos processos de trabalho, para a otimização das SO e assistência de qualidade ao paciente no período perioperatório ${ }^{8}$.

A ausência de indicadores de eficiência e da taxa de produtividade do CC foram limitações para o estudo ora em questão, uma vez que não há referência para comparar os dados coletados e o preenchimento incorreto dos dados nos impressos, dificultando sua estratificação.

\section{CONCLUSÃO}

Os indicadores gerenciais analisados neste estudo foram de produtividade cirúrgica e de cancelamento de cirurgias e suas causas, pois eles se destacam como indicadores de desempenho. Observaram-se aspectos organizacionais, como cirurgias por sala e por especialidades, porte cirúrgico e serviços de apoio solicitados no mapa cirúrgico.

A produtividade cirúrgica identificada configura-se conforme a realidade de hospitais-escola na média de atendimentos realizados.

Verificou-se semelhança na taxa de cancelamento de outras realidades de HU neste estudo, e as causas de cancelamento de maior frequência são inerentes ao planejamento, demonstrando ineficiência.

No mapa cirúrgico há falta de informações, que geram imprevisibilidade, contribuindo para a taxa de cancelamento e baixa produção, considerando a taxa de 1,3 cirurgia por SO funcional.

O perfil de atendimento do HU deste estudo pôde ser percebido e delineado pela realização dos projetos, o que determinou a frequência do porte II como a mais recorrente. Sugere-se como estratégia para diminuir a taxa de cancelamento cirúrgico e, por conseguinte, aumentar a produtividade cirúrgica o uso do bate-mapa como uma ferramenta gerencial que visa identificar os possíveis problemas relacionados à suspensão da cirurgia, como também a visita pré-operatória, além da organização do planejamento por porte cirúrgico e controle dos recursos humanos, materiais/equipamentos da assistência transoperatória.

Espera-se que o presente estudo contribua para percepção e discussão dos indicadores de desempenho do CC, especialmente de HU, de maneira a acrescentar ações destinadas ao melhor planejamento de recursos e como estratégias de otimização na gestão dos recursos totais a uma assistência de qualidade prestadas tanto pelo enfermeiro perioperatório quanto pelo gestor desse serviço.

Cabe dizer que os fatores limitantes deste estudo foram baseados na qualidade do preenchimento dos impressos, na falta de informatização dos dados, na impossibilidade de averiguação de situações diagnósticas para casos de urgência, a fim de melhorar a fidedignidade das condições apresentadas.

Recomenda-se que mais estudos ocorram, visando obter taxas de produtividade de CC e seus indicadores de eficiência, para, assim, enriquecer o tema, por meio de comparações de dados e resultados. 


\section{REFERÊNCIAS}

1. Carvalho R, Bianchi ERF. Enfermagem em centro cirúrgico e recuperação. 2a ed. São Paulo: Manole; 2016.

2. Associação Brasileira de Enfermeiros de Centro Cirúrgico, Recuperação Anestésica e Centro de Material e Esterilização. Diretrizes de práticas em enfermagem cirúrgica e processamento de produtos para a saúde. $7^{\mathrm{a}}$ ed. Barueri: Manole; São Paulo: SOBECC; 2017.

3. Association of periOperative Registered Nurses. Categoria dos padrões administrativos para enfermagem perioperatória - parte I. Rev SOBECC. 2002;7(2):8-10.

4. Brasil. Agência Nacional de Vigilância Sanitária. RDC n 50 , de 21 de fevereiro de 2002. Dispõe sobre o Regulamento Técnico para planejamento, programação, elaboração e avaliação de projetos físicos de estabelecimentos assistenciais de saúde. Brasília: Diário Oficial da União; 2002.

5. Possari JF, Gaidzinski RR, Lima AFC, Fugulin FMT, Herdman TH. Use of the nursing intervention classification for identifying the workload of a nursing team in a surgical center. Rev Latino-Am Enfermagem. 2015;23(5):781-8. http://doi.org/10.1590/0104-1169.0419.2615

6. Bonacim CAG, de Araujo AMP. Gestão de custos aplicada a hospitais universitários públicos: a experiência do Hospital das Clínicas da Faculdade de Medicina de Ribeirão Preto da USP. Rev Adm Pública [Internet]. 2010 [acessado em 22 julho 2018];44(4):903-1. Disponível em: http://www.scielo.br/pdf/rap/ v44n4/v44n4a07.pdf.

7. Duarte IG, Ferreira DP. Use of indicators in surgical center management. Rev Adm Saúde. 2006;8(31):63-70.

8. Camilo MB, Campos LI, Viana SMN, Camargos MCS, Villa EA, Zocratto KBF. Reasons for cancellations, delays and replacement of elective surgeries in a university hospital in Minas Gerais. Rev Acred. 2017;7(13):1-11.

9. Botazini NO, de Carvalho R. Cancellation of surgeries: an integrative literature review. Rev SOBECC. 2017;22(4):230-44. http://doi. org/10.5327/Z1414-4425201700040008

10. Carvalho TA, Sobral CB, Marinho PML, Llapa-Rodriguez EOO, de Aguiar Campos MP. Suspension of surgery at a university hospital. Rev SOBECC. 2016;21(4):186-91. http://doi.org/10.5327/ Z1414-4425201600040002
11. Pinheiro SL, Vasconcelos RO, de Oliveira JLC, de Oliveira Azevedo Matos FG, Tonini NS, Alves DCl. Surgical cancellation rate: quality indicator at a public university hospital. Rev Mineira Enferm. 2017;21 .

12. Moraes PGS, Pacheco NMD, Silva RGS, Silva PCV. Clinical and organizational factors related to cancellation of surgical procedures. Rev Enferm UFPE On-line. 2017;11(7):2645-53. https://doi. org/10.5205/1981-8963-v11i7a23436p2645-2653-2017

13. Santos GAAC, Bocchi SCM. Cancellation of elective surgeries in a Brazilian public hospital: reasons and estimated reduction. Rev Bras Enferm. 2017;70(3):561-8. https://doi.org/10.1590/0034-7167-2016-0084

14. Nascimento LA, Fonseca LF, Garcia ACKA. Deferral of surgery: the perspective of the medical resident in surgical clinics. Rev Bras Educ Méd. 2014;38(2):205-12. http://dx.doi.org/10.1590/\$0100-55022014000200007

15. Stroparo JR. Utilização da modelagem e simulação de sistemas na melhoria da eficiência operacional de centros cirúrgicos [dissertação]. Curitiba: Programa de Pós-Graduação em Tecnologia da Saúde, Pontífice Universidade Católica do Paraná; 2005.

16. Heiser R. Using a best-practice perioperative governance structure to implement better block scheduling. AORN J. 2013;97(1):125-31. https://doi.org/10.1016/j.aorn.2012.10.007

17. Martins DB, Portulhak H, Voese SB. Cost management: a diagnosis in federal university hospital. Rev Adm Hospitalar Inovação Saúde. 2015;12(3):59-75. https://doi.org/10.21450/rahis.v12i3.2461

18. Tamiasso RSS, Santos DC, Fernandes VDO, loshida CAF, Poveda VB, Turrini RNT. Quality management tools as strategies for reducing surgery cancellations and delays. Rev SOBECC. 2018;23(2):96-102. https://doi.org/10.5327/Z1414-4425201800020007

19. Pedro DRC, Oliveira JLC, Tonini N, Oliveira Azevedo Matos FG, Nicola AL. Dimensioning of nursing staff in a surgical center of a university hospital. J Nurs Health. 2018;8(1):e188108.

20. Gomes LC, Dutra KE, Pereira ALS. 0 enfermeiro no gerenciamento do centro cirúrgico. Rev Eletron Faculdade Metodista Granbery [Internet]. 2014 [acessado em 25 de julho de 2018];(16). Disponível em: http://re.granbery.edu.br/artigos/NTEy.pdf

21. Possari JF. Centro cirúrgico: planejamento, organização e gestão. 5a ed. São Paulo: látria; 2011. 